\title{
Curcumin, A Polyphenolic Curcuminoid With Its Protective Effects and Molecular Mechanisms in Diabetes and Diabetic Cardiomyopathy
}

\author{
Jia Zheng ${ }^{1 t}$, Jing Cheng ${ }^{2 t}$, Sheng Zheng ${ }^{3 t}$, Qianyun Feng ${ }^{3}$ and Xinhua Xiao ${ }^{1 *}$ \\ 1 Department of Endocrinology, Key Laboratory of Endocrinology, Ministry of Health, Peking Union Medical College Hospital, \\ Diabetes Research Center of Chinese Academy of Medical Sciences and Peking Union Medical College, Beijing, China, \\ ${ }^{2}$ The Key Laboratory of Cardiovascular Remodeling and Function Research, Chinese Ministry of Education and Chinese \\ Ministry of Health, The State and Shandong Province Joint Key Laboratory of Translational Cardiovascular Medicine, \\ Shandong University Qilu Hospital, Jinan, China, ${ }^{3}$ Graduate School, Tianjin University of Traditional Chinese Medicine, \\ Tianjin, China
}

OPEN ACCESS

Edited by:

Changhua Wang,

Wuhan University, China

Reviewed by:

Nazareno Paolocci, Johns Hopkins University,

United States

Graziamaria Corbi,

University of Molise, Italy

${ }^{*}$ Correspondence:

Xinhua Xiao

xiaoxh2014@vip.163.com

${ }^{\dagger}$ These authors have contributed equally to this work.

Specialty section:

This article was submitted to Cardiovascular and Smooth Muscle

Pharmacology,

a section of the journal

Frontiers in Pharmacology

Received: 02 February 2018

Accepted: 23 April 2018

Published: 09 May 2018

Citation:

Zheng J, Cheng J, Zheng S, Feng Q and Xiao X (2018) Curcumin,

A Polyphenolic Curcuminoid With Its

Protective Effects and Molecular Mechanisms in Diabetes and Diabetic

Cardiomyopathy.

Front. Pharmacol. 9:472.

doi: 10.3389/fphar.2018.00472
As the leading cause of morbidity and mortality in patients with diabetes, diabetic cardiomyopathy (DCM) imposes enormous burden on individuals and public health. Therapeutic regimes for DCM treatment have proven to be challenging, with limited efficacy, low compliance, and potential adverse effects. Curcumin, as the most active compound derived from the root of turmeric, exhibits strong anti-inflammation, antioxidant, and anti-apoptosis properties. Recently, clinical trials and preclinical studies have shown that curcumin exerts protective effects against a variety of diseases, including diabetes and its cardiovascular complications. In this review, the clinical trials about curcumin supplementation on diabetes and DCM are presented, and the specific mechanisms by which curcumin might mitigate diabetes and DCM are fully discussed. A better understanding of the pharmacological role of curcumin on diabetes and DCM can provide clinical implications for the intervention of the onset and development of diabetes and DCM.

Keywords: curcumin, curcuminoids, diabetes mellitus, diabetic cardiomyopathy, inflammation, antioxidant, apoptosis

\section{INTRODUCTION}

The prevalence of diabetes is increasing rapidly during the last three decades, which is becoming one of the most epidemic non-communicable diseases. The International Diabetes Federation [IDF] (2017) estimates that the number of people with diabetes reaches 425 million worldwide in 2017 and it will rise to 629 million by 2045, indicating a 45\% increase throughout the world. Globally, about one in eleven adults have diabetes, and $90 \%$ of whom are diagnosed as type 2 diabetes mellitus (T2DM). T2DM and its complications have contributed tremendously to the burden of mortality and health cost worldwide. Among the various complications of diabetes, cardiovascular complications are believed to be the leading causes of disability and death among diabetic patients, particularly for diabetic cardiomyopathy (DCM) (Cai and Kang, 2003). Cardiovascular diseases (CVDs) typically develop about 15 years earlier in T2DM 
(Booth et al., 2006), and T2DM patients are more than twice as likely to develop CVDs as compared with those without T2DM (Sarwar et al., 2010).

\section{LIMITATIONS IN THERAPEUTIC STRATEGIES FOR DIABETES AND DCM}

In 2016, the Global Burden of Diseases (GBD) study reported that T2DM and its complications accounted for a $22 \%$ increase in disability-adjusted life years (DALYs) during the last decade (2016), imposing enormous burden on individuals and public health (GBD 2015 Risk Factors Collaborators, 2016). Despite prominent advances in diabetes prevention, treatment, glucose monitoring and novel glycemic control biomarkers, detrimental cardiovascular complications, especially for DCM still remain rigorous in patients with T2DM (Pirola et al., 2010). Nowadays, therapeutic regimes for diabetes and DCM include several clinical managements, involving lifestyle modifications (diet and exercise), glucose and lipid control (antidiabetic and lipid-lowering drugs), hypertension treatment, and coronary artery diseases intervention. The commonly used therapeutic strategies for CVDs in diabetic patients include cardiac glycoside, $\mathrm{Ca}^{2+}$ antagonist, $\beta$-adrenergic blocking agents, angiotensin converting enzyme inhibitor (ACEI), angiotensin receptor blocker (ARB) and diuretics (Marwick et al., 2018). However, the incidence and mortality rate of DCM still remains high, it is imperative to develop novel and effective therapeutic strategies for diabetes and DCM.

\section{CURCUMIN AND ITS PROTECTIVE EFFECTS ON HUMAN HEALTH}

Plants and herbs have historically been widely used for medicinal purposes. Traditional Chinese Medicine (TCM), with a history of more than 2,000 years, includes various forms of herbal medicine and dietary therapy (Hao et al., 2015b). Natural plants and their active derivatives have been deemed as novel therapeutic agents for multiple diseases, such as CVDs (Hao et al., 2017), metabolic disorders (Martel et al., 2017), rheumatic autoimmune diseases (Dahan et al., 2017), and cancer (Seca and Pinto, 2018). A randomized, placebocontrolled study indicated that dietary supplementation with equol and resveratrol can reduce the severity of menopausal symptoms in recently postmenopausal women, that can improve menopause-related quality of life in healthy women (Davinelli et al., 2017). Curcumin, a natural compound, is the most active agent of the polyphenolic curcuminoids derived from the root of turmeric (Curcuma longa). It is a tautomeric compound existing in organic solvents as its enolic form, and in water as a keto form (Manolova et al., 2014) (Figure 1). Traditionally, turmeric, as a member of the ginger family, has been widely used as an herbal medicine, ingredient of cosmetics, and dietary supplement (food flavoring and coloring).
In addition to a dietary ingredient, turmeric is also prescribed abundantly for ailments in traditional medicine (Nelson et al., 2017). Numerous studies suggest that curcumin is a potent molecule that can exert a variety of positive pharmacological effects, including anti-inflammation (Sikora et al., 2010; Koeberle and Werz, 2014), antioxidant (Nakmareong et al., 2011), and anti-apoptosis (Topcu-Tarladacalisir et al., 2013) properties. Corbi et al. (2016) also showed that curcumin can exert relevant immunomodulatory and/or anti-inflammatory activities in the context of brain aging. Some phytochemicals, such as curcumin inducing increase in nuclear factor (erythroidderived 2)-like 2 (Nrf2) and sirtuin 1 (SIRT1) activity could be able to inhibit the nuclear factor kappa-B (NF-kB) activation and then to end the progression of the brain aging (Corbi et al., 2016). In recent years, compelling data indicates that curcumin is a protective compound against insulin resistance (Yekollu et al., 2011), obesity (Hariri and Haghighatdoost, 2018), diabetes mellitus (Arivazhagan et al., 2015), and CVDs (Hashemzaei et al., 2017; Jiang et al., 2017). However, although the evidence alludes to protective effects of curcumin on human health, information about the effect of curcumin on diabetes and DCM is limited. It is speculated that curcumin may be a pleiotropic molecule targeting diabetes and DCM, with a rather diverse array of metabolic, cellular, and molecular activities. Therefore, the current review aimed to provide an overview of the effect of curcumin on diabetes and DCM, and the molecular mechanisms of curcumin in alleviating diabetes and DCM.

\section{MOLECULAR MECHANISMS UNDERLYING THE PATHOGENESIS OF DIABETES AND DCM}

Diabetic cardiomyopathy, as a severe complication of diabetes, is characterized by cardiac structure and function disorders (Bugger and Abel, 2014), including metabolic deregulation, left ventricular dysfunction, and myocardial cell deterioration (Marwick et al., 2018). DCM is associated with impaired systolic and diastolic functions with prolonged contraction and relaxation duration, and depressed myocardial contractility and relaxation (Boudina and Abel, 2007). Echocardiography revealed shorter left ventricular ejection duration, and increased wall stiffness in diabetic patients (Poirier et al., 2001). The pathogenesis of diabetes and DCM is multifactorial, and evidence indicates that the risks of diabetes and DCM are not limited to traditional factors, such as atherosclerosis, hypertension, and coronary diseases (Boudina and Abel, 2007). To date, a range of molecular and cellular mechanisms have been proposed for the development and progress of diabetes and DCM, including advanced glycation end products (AGEs) accumulation (Goldin et al., 2006), inflammation activation (Diamant et al., 2005), increased oxidative stress (Anderson et al., 2009), higher induction of apoptosis (Frustaci et al., 2000), and impaired autophagy (Nakai et al., 2007). 


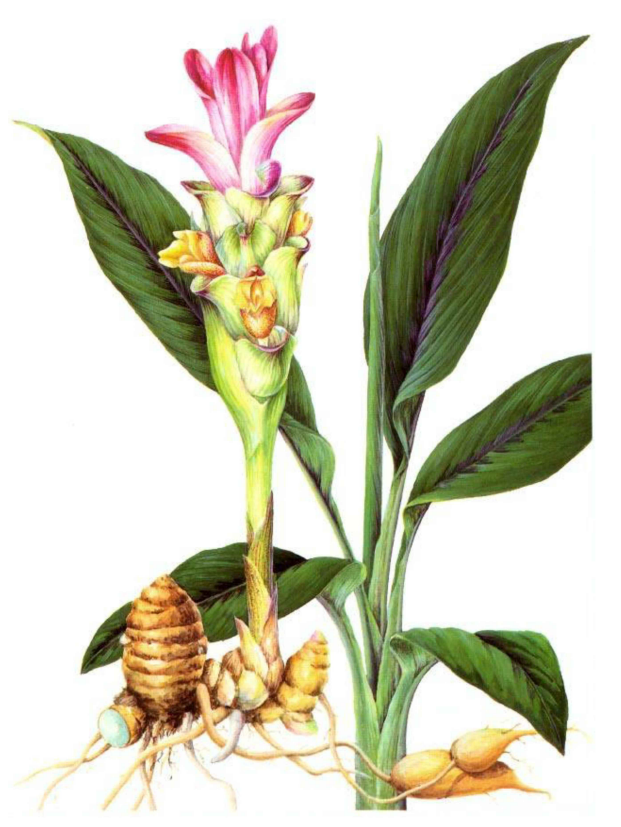<smiles>COc1cc(/C=C/C(=O)/C=C(O)/C=C/c2ccc(O)c(OC)c2)ccc1O</smiles><smiles>COc1cc(/C=C/C(=O)CC(=O)/C=C/c2ccc(O)c(OC)c2)ccc1O</smiles>

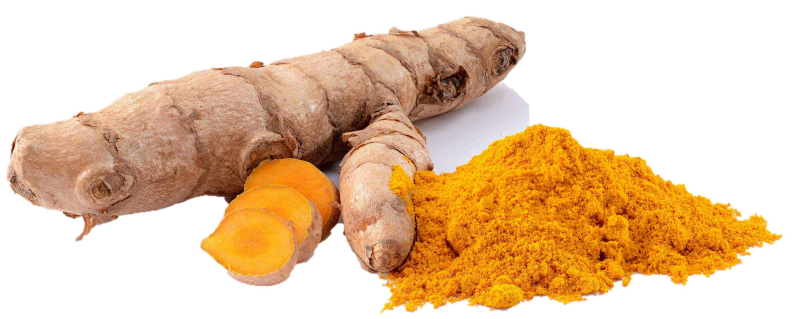

FIGURE 1 | The molecular structure of curcumin isolated from the root of turmeric. Curcumin, a natural compound, is the most active agent of the polyphenolic curcuminoids derived from the root of turmeric (Curcuma longa). It is a tautomeric compound existing in organic solvents as its enolic form, and in water as a keto form. Turmeric, as a member of the ginger family, has been widely used as an herbal medicine, ingredient of cosmetics, and dietary supplement.

\section{CLINICAL TRIALS WITH CURCUMIN IN DIABETES AND ITS-RELATED CARDIOVASCULAR RISKS}

Accumulating clinical trials have revealed that curcumin has its beneficial effects on rheumatoid arthritis (Mande et al., 2016), inflammatory bowel disease (Taylor and Leonard, 2011), cancer (Shehzad et al., 2013; Naksuriya et al., 2014), Alzheimer's disease (Hathout et al., 2017), and other diseases (Gupta et al., 2012). However, few studies have been conducted to investigate the effects of curcumin on diabetes and itsrelated CVDs. Importantly, pharmacological research reveal that curcumin is effective, safe, and without toxicity (Prasad et al., 2014). Dyslipidemia is an established factor and increase the susceptibility of atherosclerotic heart disease in diabetic patients. Panahi et al. (2017b) aimed to examine an antiatherosclerosis effect of curcumin in diabetic patients. It showed that curcuminoids supplementation (1,000 mg/day) for 12 weeks can reduce serum levels of atherogenic lipid levels, including non-high density lipoprotein (HDL) and lipoprotein(a) $[\mathrm{Lp}(\mathrm{a})]$ in patients with T2DM (Panahi et al., 2017b). Chuengsamarn et al. (2014) reported that curcuminoids intake $(1,500 \mathrm{mg} /$ day $)$ for 6 months continuously increased insulin sensitivity, decreased pulse wave velocity, triglyceride level, and atherosclerosis incidence in patients with T2DM, indicating that curcuminoids supplementation could contribute to a lower risk of cardiovascular events in dyslipidemic patients with T2DM (Chuengsamarn et al., 2014). A report from the same group found that curcumin intervention $(1,500 \mathrm{mg} /$ day $)$ for 12 months significantly decreased the incidence of T2DM in pre-diabetic individuals, with a lower level of insulin resistance and higher adiponectin level (Chuengsamarn et al., 2012). A recent clinical trial showed that curcumin supplementation (1,000 mg/day) for 12 weeks exhibited higher adiponectin level and lower leptin concentration, with decreased leptin/adiponectin ratio (a measure of atherosclerosis) in patients with T2DM (Sahebkar et al., 2018). In addition, Panahi et al. (2017a) showed that curcuminoids supplementation (1,000 mg/day) for 8 weeks significantly increased serum total antioxidant capacity (TAC) and superoxide dismutase (SOD) activities, with reduced malondialdehyde (MDA) concentrations in diabetic patients (Panahi et al., 2017a). Since dyslipidemia, insulin resistance, and oxidative stress principally increases the risks of CVDs in diabetic patients, these studies suggest the protective role of curcumin on diabetes and its-related cardiovascular risks. However, clinical trials about the direct effects of curcumin on DCM in patients with diabetes are needed. The clinical studies about curcumin and its protective effects against diabetes and its-related cardiovascular risks are listed in Table 1.

\section{PRECLINICAL STUDIES ABOUT CURCUMIN AND ITS EFFECTS ON DIABETES AND DCM}

\section{Curcumin and Its Anti-inflammatory Effects on Diabetes and DCM}

Inflammation plays a critical role of the development of diabetes and its complications (Pereira and Alvarez-Leite, 2014), 
TABLE 1 | Clinical trials about curcumin and its protective effects against diabetes and its-related cardiovascular risks.

\begin{tabular}{|c|c|c|c|}
\hline Subjects included & Treatments & Metabolic effects & Reference \\
\hline \multirow[t]{2}{*}{118 subjects with T2DM } & Curcuminoids (1,000 mg/day) for 12 weeks & $\begin{array}{l}\text { - Reductions in serum total cholesterol, } \\
\text { non-HDL-C and Lp(a) levels }\end{array}$ & Panahi et al., 2017 \\
\hline & & - Elevations in serum HDL-C levels & \\
\hline \multirow[t]{3}{*}{240 patients with T2DM } & Curcuminoids ( $1,500 \mathrm{mg} /$ day) for 6 months & - Reduced pulse wave velocity & Chuengsamarn et al., 2014 \\
\hline & & $\begin{array}{l}\text { - Increased level of serum adiponectin and } \\
\text { decreased level of leptin }\end{array}$ & \\
\hline & & $\begin{array}{l}\text { - Reduced levels of HOMA-IR, triglyceride, } \\
\text { uric acid, visceral fat, and total body fat }\end{array}$ & \\
\hline \multirow[t]{3}{*}{240 pre-diabetic individuals } & Curcuminoids ( $1,500 \mathrm{mg} /$ day) for 12 months & $\begin{array}{l}\text { - Decreased the number of pre-diabetic } \\
\text { individuals who eventually developed T2DM }\end{array}$ & Chuengsamarn et al., 2012 \\
\hline & & $\begin{array}{l}\text { - Better overall function of } \beta \text {-cells, with higher } \\
\text { HOMA- } \beta \text { and lower C-peptide }\end{array}$ & \\
\hline & & $\begin{array}{l}\text { - A lower level of HOMA-IR and higher } \\
\text { adiponectin }\end{array}$ & \\
\hline \multirow[t]{4}{*}{118 patients with T2DM } & Curcuminoids (1,000 mg/day) for 12 weeks & - Higher adiponectin level & Sahebkar et al., 2018 \\
\hline & & - Lower leptin concentration & \\
\hline & & - Decreased leptin/adiponectin ratio & \\
\hline & & - Elevated serum ghrelin level & \\
\hline \multirow[t]{2}{*}{118 subjects with T2DM } & Curcuminoids ( 1,000 mg/day) for 8 weeks & - Elevation in serum TAC and SOD activities & Panahi et al., 2017 \\
\hline & & - Reduced MDA concentration & \\
\hline
\end{tabular}

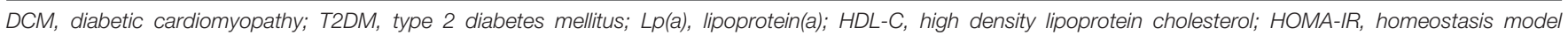
assessment-insulin resistance; HOMA- $\beta$, homeostasis model assessment- $\beta$; TAC, total antioxidant capacity; SOD, superoxide dismutase; MDA, malondialdehyde.

and inflammation with increased cytokine levels is shown to be closely related to the onset and development of diabetes and DCM (Xu et al., 2003). It demonstrated that intramyocardial inflammation in DCM is associated with increased macrophages and leucocytes infiltration, significant increment of vascular cell adhesion molecule-1 (VCAM-1) and intercellular adhesion molecule-1 (ICAM-1) expressions, as well as elevated expression of inflammatory cytokines, including interleukin-6 (IL-6), tumor necrosis factor- $\alpha$ (TNF- $\alpha$ ), interleukin-1 $\beta$ (IL$1 \beta$ ), interleukin-18 (IL-18), and transforming growth factor- $\beta 1$ (TGF- $\beta 1$ ) (Tschope et al., 2005; Westermann et al., 2007; Rajesh et al., 2012; You et al., 2018). Recent evidence reveals that nucleotide-binding oligomerization domain like receptor (NLR) pyrin domain containing 3 (NLRP3) inflammasome is also a promising molecular marker of diabetes and DCM (Luo et al., 2017).

Curcumin is a potent anti-inflammatory agent and considered beneficial for the amelioration of diabetes and DCM. Curcumin was able to suppress NF- $\kappa \mathrm{B}$ signaling pathway, defend against inflammation, cardiac hypertrophy and fibrosis in the heart (He et al., 2015). Curcumin supplementation decreased serum inflammatory factors levels of IL-6, TNF- $\alpha$ and monocyte chemoattractant protein-1 (MCP-1), as well as glucose and glycosylated hemoglobin in diabetic rats. Curcumin incubation also inhibited IL-6, IL-8, TNF- $\alpha$, and MCP-1 secretion in high glucose-treated monocytes (Jain et al., 2009). Yu et al. (2012) showed that curcumin inhibited AGEs accumulation, decreased inflammatory cytokines of IL- $1 \beta$ and TNF- $\alpha$, and attenuated diabetes-induced left ventricular dysfunction, cardiomyocyte hypertrophy and interstitial fibrosis in diabetic rats. A similar study reported that curcumin dramatically decreased IL-6 and TNF- $\alpha$ levels in streptozotocin-induced diabetic rats with heart injury (Abo-Salem et al., 2014). A recent study showed that curcumin (300 mg/kg/day for 16 weeks) significantly suppressed collagens deposition in diabetic rat heart, with marked reduction of TGF- $\beta 1$ production, suppression of type II TGF- $\beta$ (T $\beta$ RII) levels and sma- and mad- related protein $2 / 3$ (Smad2/3) phosphorylation, and increment of Smad7 expression in the heart of diabetic rat. They further found that application of curcumin inhibited TGF- $\beta 1$ - or high glucose-induced adenosine monophosphate activated protein kinase (AMPK)/p38 mitogen-activated protein kinase (MAPK) activation in human cardiac fibroblasts in vitro (Guo et al., 2018).

In recent years, curcumin analog has been discovered and widely investigated for their roles in diabetes and DCM. A novel curcumin analog C66 reduces serum and heart hypertriglyceridemia, accompanied by improved cardiac function, inhibition of Jun NH2-terminal kinase (JNK) signaling and cardiac inflammation. They further reported that curcumin analog C66 inhibited a high glucose-induced rise in pro-inflammatory cytokines via inactivation of NF- $\mathrm{B}$ (Wang et al., 2014). J17, another molecule with structural similarities to curcumin, exerts significant inhibitory effects on hyperglycemia-induced inflammation and fibrosis in H9C2 cardiomyocytes and streptozotocin-induced diabetic mouse. The underlying mechanisms may be associated with the inhibition of the P38 and protein kinase B (AKT) signal pathway (Chen et al., 2017). Thus, these studied indicated that curcumin and its analogs can alleviate DCM by attenuating inflammation in vivo and in vitro. The relevant evidence is summarized in Table 2 , and the potential mechanism by which 
TABLE 2 | Pre-clinical studies about curcumin and its effects on diabetes and DCM.

\section{Animals/cells \\ Anti-inflammatory effects}

STZ-induced diabetic SD rats

High glucose-treated monocytes STZ-induced diabetic Wistar rats

STZ-induced diabetic Wistar rats STZ-induced diabetic SD rats

High glucose-treated human cardiac fibroblasts

STZ-induced diabetic C57BL/6 mice

STZ-induced diabetic C57BL/6 mice

High glucose-treated H9C2 cardiomyocytes

\section{Antioxidant properties}

STZ-induced diabetic Wistar rats

STZ-induced diabetic Wistar rats

STZ-induced diabetic SD rats

STZ-induced diabetic C57BL/6 mice

STZ-induced diabetic rats

\section{Treatments}

Curcumin (100 mg/kg/day) for 7 weeks

Curcumin incubation $(0.01-1 \mu \mathrm{M})$ for $24 \mathrm{~h}$

Curcumin (100 or 200 mg/kg/day) for 8 weeks

Curcumin (200 mg/kg/day) for 6 weeks

Curcumin (300 mg/kg/day) for 16 weeks

Curcumin incubation $(25 \mu \mathrm{M})$ for $24 \mathrm{~h}$

Curcumin (5 mg/kg/day) for 3 months

Curcumin analog, J17 (10 mg/kg/day) for 42 days

Curcumin analog, $\mathrm{J} 17(2.5$ or $10 \mu \mathrm{M})$ for $30 \mathrm{~min}$

Curcumin (100 or 200 mg/kg/day) for 8 weeks

Curcumin (200 mg/kg/day) for 6 weeks

Curcumin (100 mg/kg/day) for 8 weeks

Curcumin (5 mg/kg/day) for 3 months

Curcumin (20 mg/kg/day) for 45 days
Main findings

Reference

- Decreased blood levels of TNF- $\alpha$, IL-6, MCP-1

Jain et al., 2009

- Decreased glucose and glycosylated

hemoglobin

- Lower TNF- $\alpha$, IL-6, IL-8, and MCP-1 secretion

- Attenuated diabetes-induced left ventricular dysfunction, cardiomyocyte hypertrophy and interstitial fibrosis

- Inhibited AGEs accumulation

- Decreased inflammatory factors (TNF- $\alpha$ and

IL-1 $\beta$ )

- Activated AKT/GSK-3 $\beta$ signaling pathway

- Inhibited IL-6 and TNF- $\alpha$ levels

- Reduced TGF- $\beta 1$ production

- Suppressed T $\beta$ R II levels and Smad2/3

phosphorylation

- Increased Smad7 expression

- Inhibited TGF- $\beta 1$ - or HG-induced AMPK/p38

MAPK activation

- Suppressed collagen synthesis in the fibroblasts

- Reduced hypertriglyceridemia in both serum and hearts

- Improved cardiac function, inhibition of JNK signaling and cardiac inflammation

- Inhibited a high glucose-induced rise in pro-inflammatory cytokines via inactivation of $\mathrm{NF}-\kappa \mathrm{B}$

- Suppressed hyperglycemia-induced inflammation, hypertrophy and fibrosis

- Decreased TNF- $\alpha$ and ICAM-1

- Decreased pro-inflammatory cytokines (TNF- $\alpha$ and IL-6) and adhesion molecules (VCAM-1 and ICAM-1) expressions

- Decreased AKT phosphorylation

- Inhibited the HG-induced increase in fibrotic genes (collagen-IV, TGF- $\beta$, and collagen-I)

Attenuated NADP+/NADPH ratio, Rac1 activity and the expression of NADPH oxidase subunits of gp91 phox, p47 phox

- Restored cardiac antioxidant enzymes (catalase, superoxide dismutase, and glutathione-S-transferase)

- Decreased NADPH oxidase subunits (p67phox, p22phox, gp91phox)

- Decreased the mRNA expression of transcriptional co-activator p300 and atrial natriuretic peptide

- Decreased accumulation of ECM protein

- Reversed the increment of superoxide production

- Protection against diabetes-induced cardiac fibrosis, oxidative stress, and ER

- Prevented diabetes-induced upregulation of $\mathrm{HO}-1$ expression and activity
Jain et al., 2009

Yu et al., 2012

Abo-Salem et al., 2014

Guo et al., 2018

Guo et al., 2018

Wang et al., 2014

Chen et al., 2017

Chen et al., 2017

Yu et al., 2012

Abo-Salem et al., 2014

Soetikno et al., 2012

Wang et al., 2014

Aziz et al., 2013 
TABLE 2 | Continued

\section{Animals/cells}

Treatments

Main findings

Reference

Anti-apoptotic effects

STZ-induced diabetic Wistar rats

Curcumin (100 or 200 mg/kg/day) for 8 weeks

Prevented diabetes-induced cardiomyocytes apoptosis

STZ-induced diabetic C57BL/6 mice

Curcumin (5 mg/kg/day) for 8 weeks

- Prevented high glucose-induced apoptosis in cardiomyocytes and the development of diabetic cardiomyopathy

- Inhibition of JNK phosphorylation

\section{STZ-induced diabetic C57BL/6 mice} cardiomyocytes
High glucose-treated neonatal rat
Curcumin (5 mg/kg/day) for 3 months

Curcumin incubation (10 $\mu \mathrm{M})$ for $24 \mathrm{~h}$
Protection against diabetes-induced cardiac fibrosis, oxidative stress, and ER;

- Inhibited the increased $\mathrm{Bax} / \mathrm{Bcl}-2$ ratio elicited by high glucose exposure
Yu et al., 2012

Pan et al., 2014

Wang et al., 2014

Yu et al., 2016
- Increased AKT and GSK-3 $\beta$ phosphorylation

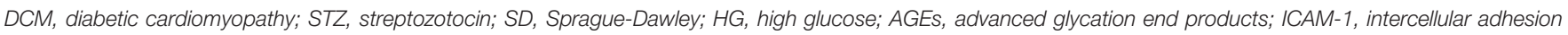
molecule-1; VCAM-1, vascular cell adhesion molecule-1; IL-1 $\beta$, interleukin-1 $\beta$; IL-6, interleukin-6; IL-18, interleukin-18; TNF- $\alpha$, tumor necrosis factor- $\alpha$; TGF- $\beta 1$,

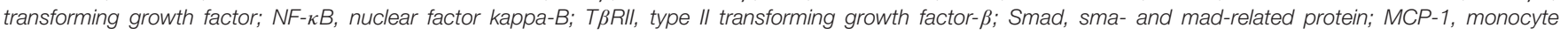
chemoattractant protein-1; AMPK, adenosine monophosphate activated protein kinase; MAPK, mitogen-activated protein kinase; JNK, Jun NH2-terminal kinase;

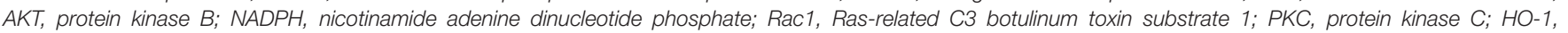
heme-oxygenase-1; GSK-3 $\beta$, glycogen synthase kinase 3 $\beta$; ECM, extracellular matrix; ER, endoplasmic reticulum.

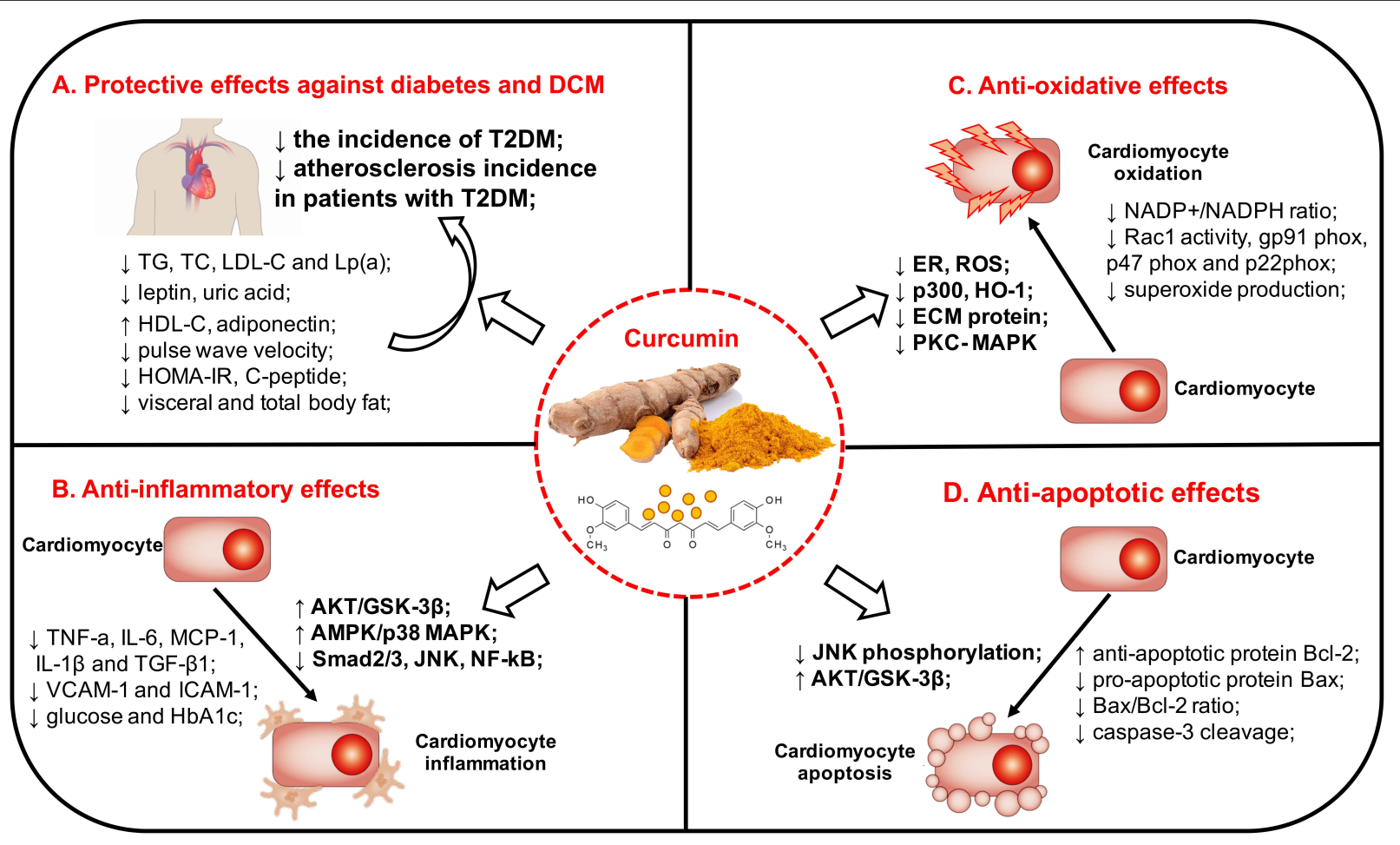

FIGURE 2 | The clinical evidence and potential mechanism by which curcumin might mitigate diabetes and DCM. The pathogenesis of diabetes and DCM is multifactorial, and a plethora of molecular mechanisms have been postulated for the onset and development of diabetes and DCM. Clinical trials have shown that curcumin possessed a potency to decrease blood glucose, improve insulin resistance and ameliorate dyslipidemia in patients with diabetes. Curcumin also exerts a variety of positive effects that was able to attenuate inflammatory activation, oxidative stress, and apoptosis in diabetes and DCM through several signaling pathways. DCM, diabetic cardiomyopathy; T2DM, type 2 diabetes mellitus; DCM, diabetic cardiomyopathy; LDL-C, low density lipoprotein cholesterol; Lp(a), lipoprotein(a); HDL-C, high density lipoprotein cholesterol; HOMA-IR, homeostasis model assessment-insulin resistance; IL-1 $\beta$, interleukin-1 $\beta$; IL-6, interleukin-6; IL-18, interleukin-18; TNF- $\alpha$, tumor necrosis factor- $\alpha$; TGF- $\beta 1$, transforming growth factor; ICAM-1, intercellular adhesion molecule-1; VCAM-1, vascular cell

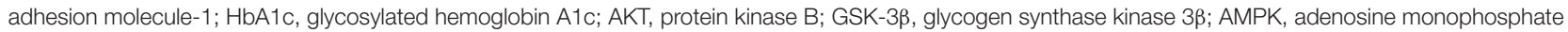
activated protein kinase; MAPK, mitogen-activated protein kinase; Smad, sma- and mad- related protein; JNK, Jun NH2-terminal kinase; NF-кB, nuclear factor kappa-B; T $\beta$ RII, type II transforming growth factor- $\beta$; MCP-1, monocyte chemoattractant protein-1; NADPH, nicotinamide adenine dinucleotide phosphate; Rac1, Ras-related C3 botulinum toxin substrate 1; HO-1, heme-oxygenase-1; ER, endoplasmic reticulum; ROS, reactive oxygen species; ECM, extracellular matrix; PKC, protein kinase C. (A) Curcumin and its protective effects of against diabetes and DCM. (B) Curcumin and its anti-inflammatory effects on diabetes and DCM. (C) Curcumin and its antioxidant properties on diabetes and DCM. (D) Curcumin and its anti-apoptotic effects on diabetes and DCM. 
curcumin might mitigate diabetes and DCM is exhibited in Figure 2.

\section{Curcumin and Its Antioxidant Properties on Diabetes and DCM}

Oxidative stress is known to participate in the development of diabetes and DCM. Reactive oxygen species (ROS) have been postulated to play a significant role in the pathogenesis of diabetes and DCM. Anderson et al. firstly showed the evidence of increased oxidative stress, with higher mitochondrial hydrogen peroxide $\left(\mathrm{H}_{2} \mathrm{O}_{2}\right)$ production and elevated 4-hydroxynonenaland 3-nitrotyrosine-modified proteins levels in atrial tissue in human diabetic hearts (Anderson et al., 2009). Furthermore, Ye et al. (2004) provided the evidence of causality between ROS and DCM. It indicated that overexpression of catalase or manganese SOD partially restored the dysfunction of mitochondrion and cardiomyocyte contractility in diabetic mice.

Curcumin was able to act as a natural free radical scavenger as its chemical structure and has anti-oxidative potency. Abo-Salem et al. (2014) found that curcumin mitigated the reduction of cardiac antioxidant enzymes and glutathione levels in diabetic rats, including SOD, catalase, and glutathione-S-transferase. It showed that curcumin attenuated nicotinamide adenine dinucleotide phosphate (NADPH) oxidase subunits (p47phox and gp91phox) expressions, NADP+/NADPH ratio, and Ras-related C3 botulinum toxin substrate 1 (Rac1) activity in diabetic rats (Yu et al., 2012). Soetikno et al. (2012) also showed that curcumin treatment markedly decreased NADPH oxidase subunits (p67phox, p22phox, gp91phox) expressions and superoxide production in diabetic rats, possibly by inhibiting protein kinase C (PKC)- MAPK signaling pathway. The novel curcumin analog C66 was able to inhibit JNK activation in diabetes, resulting in protection of endoplasmic reticulum (ER) and oxidative stress against induced by diabetes (Wang et al., 2014). Aziz et al. (2013) reported that curcumin protected against diabetes-induced hemeoxygenase-1 (HO-1) upregulation in the cardiac tissue of diabetic rats.

In addition, the heart depends on continuous mitochondrial ATP supply and maintained redox balance to properly develop force. However, myocardial energetic-redox balance is perturbed exposed to hyperglycemia, that is a critical driver of mitochondrial dysfunction in the diabetic myocardium (Aon et al., 2015). Mitochondria control cell respiration and energy production are also closely related to oxidative stress. Wright et al. (2011) found reduced cardiac function and increased myocardial oxygen consumption in $d b / d b$ diabetic mice, that was possibly regulated by increased mitochondrial ROS generation and lipid and protein peroxidation products in mice hearts. Alleviating oxidative stress targeting mitochondrial function has been confirmed as a potential therapeutic approach to limit ischemia-reperfusion (IR)-induced cardiac injury and protect against diabetes (Yang et al., 2013). Thus, preserving mitochondrial function, especially maintaining mitochondrial redox potential is also an important mechanism underlying the protective effects of curcumin against oxidative stress and diabetes. It showed that curcumin significantly decreased palmitate-induced oxidative stress and increased mitochondrial permeability, thus it was able to promote glucose-induced insulin secretion in pancreatic $\beta$-cells (Hao et al., 2015a). Lin et al. (2014) showed that collagen fibers in interstitium and expansion of mitochondria in cytoplasm of myocardium were increased in diabetic rats, that can be ameliorated by curcumin derivative B06 treatment. However, studies about direct effects targeting mitochondrial function of curcumin against DCM are limited, and further investigations about curcumin and its role in preserving mitochondrial function in DCM are needed. Thus, these studied indicated that curcumin can play its antioxidant properties in diabetes and DCM (Table 2 and Figure 2).

\section{Curcumin and Its Anti-apoptotic Effects on Diabetes and DCM}

Higher degree of cell apoptosis can be observed in the hearts of diabetic patients and in rodent models of diabetes. It has been shown that renin-angiotensin-aldosterone system (RAAS) activation, increased production of ROS, and abnormal expressions of apoptosis-related molecules are all associated with cardiomyocyte apoptosis and necrosis in diabetic hearts (Frustaci et al., 2000). Yu et al. (2012) found that curcumin supplementation (200 $\mathrm{mg} / \mathrm{kg} /$ day $)$ for 8 weeks prevented DM-induced cardiomyocytes apoptosis, evaluated by increased TUNEL-positive cells in diabetic rats. Pan et al. (2014) demonstrated that curcumin analog mitigated high glucose-induced apoptosis in cardiomyocytes and prevented the development of DCM, through the inhibition of JNK phosphorylation in the diabetic heart (Ren and Sowers, 2014). Wang et al. (2014) indicated a marked decrease in anti-apoptotic protein (Bcl-2), as well as an increase in caspase-3 cleavage and pro-apoptotic protein (Bax) in diabetic mice. However, treatment with curcumin analog $(5 \mathrm{mg} / \mathrm{kg} /$ day $)$ for 3 months significantly reversed the diabetes-induced cardiac cells apoptosis in diabetic mice (Wang et al., 2014). It also indicated that curcumin supplement effectively inhibited the elevated $\mathrm{Bax} / \mathrm{Bcl}-2$ ratio and alleviated high glucose-induced cardiomyocyte apoptosis in neonatal rat cardiomyocytes, accompanied by increased AKT and glycogen synthase kinase $3 \beta$ (GSK-3 $\beta$ ) phosphorylation (Yu et al., 2016). Thus, the evidence indicated that curcumin can alleviate cardiomyocyte apoptosis in diabetes (Table 2 and Figure 2).

\section{CONCLUSION}

In summary, as a natural compound, curcumin plays a critical role in human health. Clinical trials and preclinical studies have shown that curcumin possessed a potency to decrease blood glucose, improve insulin resistance and ameliorate dyslipidemia in patients with diabetes. It can exert positive effects on 
attenuate inflammatory activation, oxidative stress, and apoptosis in diabetes, and it is proposed to have the potential impacts to protect against DCM. Curcumin, which is believed to be pharmacologically safe, effective, and with low adverse effects, it can be considered as a promising agent for alternative therapies for the prevention and treatment of diabetes and its cardiovascular complications. However, clinical trials about the long-term effects and precise mechanisms of curcumin on diabetes and DCM in humans are still lacking. Thus, more randomized controlled trials and preclinical experiments are required to confirm the efficacy of curcumin and to elucidate the various mechanisms by which curcumin might mitigate diabetes and DCM, which may have the potential to open new horizons in the early prevention and treatment of the development of diabetes and DCM in the future.

\section{REFERENCES}

Abo-Salem, O. M., Harisa, G. I., Ali, T. M., El-Sayed el, S. M., and Abou-Elnour, F. M. (2014). Curcumin ameliorates streptozotocin-induced heart injury in rats. J. Biochem. Mol. Toxicol. 28, 263-270. doi: 10.1002/jbt.21562

Anderson, E. J., Kypson, A. P., Rodriguez, E., Anderson, C. A., Lehr, E. J., and Neufer, P. D. (2009). Substrate-specific derangements in mitochondrial metabolism and redox balance in the atrium of the type 2 diabetic human heart. J. Am. Coll. Cardiol. 54, 1891-1898. doi: 10.1016/j.jacc.2009.07.031

Aon, M. A., Tocchetti, C. G., Bhatt, N., Paolocci, N., and Cortassa, S. (2015). Protective mechanisms of mitochondria and heart function in diabetes. Antioxid. Redox Signal. 22, 1563-1586. doi: 10.1089/ars.2014.6123

Arivazhagan, A., Krishna, S., Yadav, S., Shah, H. R., Kumar, P., and Ambasta, R. K. (2015). Synergy of bone marrow transplantation and curcumin ensue protective effects at early onset of diabetes in mice. J Diabetes 7, 473-484. doi: 10.1111/1753-0407.12204

Aziz, M. T., El Ibrashy, I. N., Mikhailidis, D. P., Rezq, A. M., Wassef, M. A., Fouad, H. H., et al. (2013). Signaling mechanisms of a water soluble curcumin derivative in experimental type 1 diabetes with cardiomyopathy. Diabetol. Metab. Syndr. 5:13. doi: 10.1186/1758-5996-5-13

Booth, G. L., Kapral, M. K., Fung, K., and Tu, J. V. (2006). Relation between age and cardiovascular disease in men and women with diabetes compared with non-diabetic people: a population-based retrospective cohort study. Lancet 368, 29-36. doi: 10.1016/S0140-6736(06)68967-8

Boudina, S., and Abel, E. D. (2007). Diabetic cardiomyopathy revisited. Circulation 115, 3213-3223. doi: 10.1161/CIRCULATIONAHA.106.679597

Bugger, H., and Abel, E. D. (2014). Molecular mechanisms of diabetic cardiomyopathy. Diabetologia 57, 660-671. doi: 10.1007/s00125-014-3171-6

Cai, L., and Kang, Y. J. (2003). Cell death and diabetic cardiomyopathy. Cardiovasc. Toxicol. 3, 219-228. doi: 10.1385/CT:3:3:219

Chen, H., Yang, X., Lu, K., Lu, C., Zhao, Y., Zheng, S., et al. (2017). Inhibition of high glucose-induced inflammation and fibrosis by a novel curcumin derivative prevents renal and heart injury in diabetic mice. Toxicol. Lett. 278, 48-58. doi: 10.1016/j.toxlet.2017.07.212

Chuengsamarn, S., Rattanamongkolgul, S., Luechapudiporn, R., Phisalaphong, C., and Jirawatnotai, S. (2012). Curcumin extract for prevention of type 2 diabetes. Diabetes Care 35, 2121-2127. doi: 10.2337/dc12-0116

Chuengsamarn, S., Rattanamongkolgul, S., Phonrat, B., Tungtrongchitr, R., and Jirawatnotai, S. (2014). Reduction of atherogenic risk in patients with type 2 diabetes by curcuminoid extract: a randomized controlled trial. J. Nutr. Biochem. 25, 144-150. doi: 10.1016/j.jnutbio.2013.09.013

Corbi, G., Conti, V., Davinelli, S., Scapagnini, G., Filippelli, A., and Ferrara, N. (2016). Dietary phytochemicals in neuroimmunoaging: a new therapeutic possibility for humans? Front. Pharmacol. 7:364. doi: 10.3389/fphar.2016.00364

Dahan, S., Segal, Y., and Shoenfeld, Y. (2017). Dietary factors in rheumatic autoimmune diseases: a recipe for therapy? Nat. Rev. Rheumatol. 13, 348-358. doi: $10.1038 /$ nrrheum.2017.42

\section{AUTHOR CONTRIBUTIONS}

JZ and JC collected and synthesized the data and wrote the manuscript. QF and SZ reviewed and edited the manuscript. JZ and XX contributed to the design of this review.

\section{FUNDING}

This study was sponsored by National Key R\&D Program of China (2017YFC1309603), National Key Research and Development Program of China (2016YFA0101002), National Natural Science Foundation of China (No. 81170736 and 81570715), Beijing Natural Science Foundation (No. 7184252), and the Fund for Fostering Young Scholars of Peking University Health Science Center (No. BMU2017PY008).

Davinelli, S., Scapagnini, G., Marzatico, F., Nobile, V., Ferrara, N., and Corbi, G. (2017). Influence of equol and resveratrol supplementation on health-related quality of life in menopausal women: a randomized, placebo-controlled study. Maturitas 96, 77-83. doi: 10.1016/j.maturitas.2016.11.016

Diamant, M., Lamb, H. J., Smit, J. W., De Roos, A., and Heine, R. J. (2005). Diabetic cardiomyopathy in uncomplicated type 2 diabetes is associated with the metabolic syndrome and systemic inflammation. Diabetologia 48, 1669-1670. doi: 10.1007/s00125-005-1821-4

Frustaci, A., Kajstura, J., Chimenti, C., Jakoniuk, I., Leri, A., Maseri, A., et al. (2000). Myocardial cell death in human diabetes. Circ. Res. 87, 1123-1132. doi: 10.1161/01.RES.87.12.1123

GBD 2015 Risk Factors Collaborators (2016). Global, regional, and national comparative risk assessment of 79 behavioural, environmental and occupational, and metabolic risks or clusters of risks, 1990-2015: a systematic analysis for the Global Burden of Disease Study 2015. Lancet 388, 1659-1724. doi: 10.1016/S0140-6736(16)31679-8

Goldin, A., Beckman, J. A., Schmidt, A. M., and Creager, M. A. (2006). Advanced glycation end products: sparking the development of diabetic vascular injury. Circulation 114, 597-605. doi: 10.1161/CIRCULATIONAHA.106.621854

Guo, S., Meng, X. W., Yang, X. S., Liu, X. F., Ou-Yang, C. H., and Liu, C. (2018). Curcumin administration suppresses collagen synthesis in the hearts of rats with experimental diabetes. Acta Pharmacol. Sin. 39, 195-204. doi: 10.1038/aps. 2017.92

Gupta, S. C., Patchva, S., Koh, W., and Aggarwal, B. B. (2012). Discovery of curcumin, a component of golden spice, and its miraculous biological activities. Clin. Exp. Pharmacol. Physiol. 39, 283-299. doi: 10.1111/j.1440-1681.2011. 05648.x

Hao, F., Kang, J., Cao, Y., Fan, S., Yang, H., An, Y., et al. (2015a). Curcumin attenuates palmitate-induced apoptosis in MIN6 pancreatic beta-cells through PI3K/Akt/FoxO1 and mitochondrial survival pathways. Apoptosis 20, 1420-1432. doi: 10.1007/s10495-015-1150-0

Hao, P. P., Jiang, F., Chen, Y. G., Yang, J., Zhang, K., Zhang, M. X., et al. (2015b). Traditional Chinese medication for cardiovascular disease. Nat. Rev. Cardiol. 12, 115-122. doi: 10.1038/nrcardio.2014.177

Hao, P., Jiang, F., Cheng, J., Ma, L., Zhang, Y., and Zhao, Y. (2017). Traditional Chinese Medicine for cardiovascular disease: evidence and potential mechanisms. J. Am. Coll. Cardiol. 69, 2952-2966. doi: 10.1016/j.jacc.2017. 04.041

Hariri, M., and Haghighatdoost, F. (2018). Effect of curcumin on anthropometric measures: a systematic review on randomized clinical trials. J. Am. Coll. Nutr. 37, 215-222. doi: 10.1080/07315724.2017.1392263

Hashemzaei, M., Entezari Heravi, R., Rezaee, R., Roohbakhsh, A., and Karimi, G. (2017). Regulation of autophagy by some natural products as a potential therapeutic strategy for cardiovascular disorders. Eur. J. Pharmacol. 802, 44-51. doi: 10.1016/j.ejphar.2017.02.038

Hathout, R. M., El-Ahmady, S. H., and Metwally, A. A. (2017). Curcumin or bisdemethoxycurcumin for nose-to-brain treatment of Alzheimer disease? A 
bio/chemo-informatics case study. Nat. Prod. Res. doi: 10.1080/14786419.2017. 1385017 [Epub ahead of print].

He, Y., Yue, Y., Zheng, X., Zhang, K., Chen, S., and Du, Z. (2015). Curcumin, inflammation, and chronic diseases: how are they linked? Molecules 20, 9183-9213. doi: 10.3390/molecules20059183

Jain, S. K., Rains, J., Croad, J., Larson, B., and Jones, K. (2009). Curcumin supplementation lowers TNF-alpha, IL-6, IL-8, and MCP-1 secretion in high glucose-treated cultured monocytes and blood levels of TNF-alpha, IL-6, MCP1 , glucose, and glycosylated hemoglobin in diabetic rats. Antioxid. Redox Signal. 11, 241-249. doi: 10.1089/ars.2008.2140

Jiang, S., Han, J., Li, T., Xin, Z., Ma, Z., Di, W., et al. (2017). Curcumin as a potential protective compound against cardiac diseases. Pharmacol. Res. 119, 373-383. doi: 10.1016/j.phrs.2017.03.001

Koeberle, A., and Werz, O. (2014). Multi-target approach for natural products in inflammation. Drug Discov. Today 19, 1871-1882. doi: 10.1016/j.drudis.2014. 08.006

Lin, Z. M., Jiao, L. Z., Zheng, Y., Wang, X. Y., Wang, L., Liu, W. W., et al. (2014). [The effect and mechanism of curcumin derivative B06 on the myocardium from type 2 diabetic rats]. Zhongguo Ying Yong Sheng Li Xue Za Zhi 30, 38-42.

Luo, B., Huang, F., Liu, Y., Liang, Y., Wei, Z., Ke, H., et al. (2017). NLRP3 inflammasome as a molecular marker in diabetic cardiomyopathy. Front. Physiol. 8:519. doi: 10.3389/fphys.2017.00519

Mande, P. P., Bachhav, S. S., and Devarajan, P. V. (2016). Solid dispersion of curcumin as polymeric films for bioenhancement and improved therapy of rheumatoid arthritis. Pharm. Res. 33, 1972-1987. doi: 10.1007/s11095-0161934-0

Manolova, Y., Deneva, V., Antonov, L., Drakalska, E., Momekova, D., and Lambov, N. (2014). The effect of the water on the curcumin tautomerism: a quantitative approach. Spectrochim Acta A Mol. Biomo.l Spectrosc. 132, 815-820. doi: 10.1016/j.saa.2014.05.096

Martel, J., Ojcius, D. M., Chang, C. J., Lin, C. S., Lu, C. C., Ko, Y. F., et al. (2017). Anti-obesogenic and antidiabetic effects of plants and mushrooms. Nat. Rev. Endocrinol. 13, 149-160. doi: 10.1038/nrendo.2016.142

Marwick, T. H., Ritchie, R., Shaw, J. E., and Kaye, D. (2018). Implications of underlying mechanisms for the recognition and management of diabetic cardiomyopathy. J. Am. Coll. Cardiol. 71, 339-351. doi: 10.1016/j.jacc.2017. 11.019

Nakai, A., Yamaguchi, O., Takeda, T., Higuchi, Y., Hikoso, S., Taniike, M., et al. (2007). The role of autophagy in cardiomyocytes in the basal state and in response to hemodynamic stress. Nat. Med. 13, 619-624. doi: 10.1038/ nm1574

Nakmareong, S., Kukongviriyapan, U., Pakdeechote, P., Donpunha, W., Kukongviriyapan, V., Kongyingyoes, B., et al. (2011). Antioxidant and vascular protective effects of curcumin and tetrahydrocurcumin in rats with L-NAME-induced hypertension. Naunyn Schmiedebergs Arch. Pharmacol. 383, 519-529. doi: 10.1007/s00210-011-0624-z

Naksuriya, O., Okonogi, S., Schiffelers, R. M., and Hennink, W. E. (2014). Curcumin nanoformulations: a review of pharmaceutical properties and preclinical studies and clinical data related to cancer treatment. Biomaterials 35, 3365-3383. doi: 10.1016/j.biomaterials.2013.12.090

Nelson, K. M., Dahlin, J. L., Bisson, J., Graham, J., Pauli, G. F., and Walters, M. A. (2017). The essential medicinal chemistry of curcumin. J. Med. Chem. 60, 1620-1637. doi: 10.1021/acs.jmedchem.6b00975

Pan, Y., Wang, Y., Zhao, Y., Peng, K., Li, W., Wang, Y., et al. (2014). Inhibition of JNK phosphorylation by a novel curcumin analog prevents high glucoseinduced inflammation and apoptosis in cardiomyocytes and the development of diabetic cardiomyopathy. Diabetes Metab. Res. Rev. 63, 3497-3511. doi: $10.2337 / \mathrm{db} 13-1577$

Panahi, Y., Khalili, N., Sahebi, E., Namazi, S., Karimian, M. S., Majeed, M., et al. (2017a). Antioxidant effects of curcuminoids in patients with type 2 diabetes mellitus: a randomized controlled trial. Inflammopharmacology 25, 25-31. doi: 10.1007/s10787-016-0301-4

Panahi, Y., Khalili, N., Sahebi, E., Namazi, S., Reiner, Z., Majeed, M., et al. (2017b). Curcuminoids modify lipid profile in type 2 diabetes mellitus: a randomized controlled trial. Complement. Ther. Med. 33, 1-5. doi: 10.1016/j.ctim.2017. 05.006

Pereira, S. S., and Alvarez-Leite, J. I. (2014). Low-grade inflammation, obesity, and diabetes. Curr. Obes. Rep. 3, 422-431. doi: 10.1007/s13679-014-0124-9
Pirola, L., Balcerczyk, A., Okabe, J., and El-Osta, A. (2010). Epigenetic phenomena linked to diabetic complications. Nat. Rev. Endocrinol. 6, 665-675. doi: 10.1038/ nrendo.2010.188

Poirier, P., Bogaty, P., Garneau, C., Marois, L., and Dumesnil, J. G. (2001). Diastolic dysfunction in normotensive men with well-controlled type 2 diabetes: importance of maneuvers in echocardiographic screening for preclinical diabetic cardiomyopathy. Diabetes Care 24, 5-10. doi: 10.2337/diacare.24.1.5

Prasad, S., Tyagi, A. K., and Aggarwal, B. B. (2014). Recent developments in delivery, bioavailability, absorption and metabolism of curcumin: the golden pigment from golden spice. Cancer Res. Treat. 46, 2-18. doi: 10.4143/crt.2014. 46.1.2

Rajesh, M., Batkai, S., Kechrid, M., Mukhopadhyay, P., Lee, W. S., Horvath, B., et al. (2012). Cannabinoid 1 receptor promotes cardiac dysfunction, oxidative stress, inflammation, and fibrosis in diabetic cardiomyopathy. Diabetes Metab. Res. Rev. 61, 716-727. doi: 10.2337/db11-0477

Ren, J., and Sowers, J. R. (2014). Application of a novel curcumin analog in the management of diabetic cardiomyopathy. Diabetes Metab. Res. Rev. 63, 3166-3168. doi: 10.2337/db14-0863

Sahebkar, A., Panahi, Y., Khalili, N., Sahebi, E., Namazi, S., Atkin, S. L., et al. (2018). Curcuminoids plus piperine modulate adipokines in type 2 diabetes mellitus. Curr. Clin. Pharmacol. 12, 253-258. doi: 10.2174/1574884713666180104095641

Sarwar, N., Gao, P., Seshasai, S. R., Gobin, R., Kaptoge, S., Di Angelantonio, E., et al. (2010). Diabetes mellitus, fasting blood glucose concentration, and risk of vascular disease: a collaborative meta-analysis of 102 prospective studies. Lancet 375, 2215-2222. doi: 10.1016/S0140-6736(10)60484-9

Seca, A. M. L., and Pinto, D. C. G. A. (2018). Plant secondary metabolites as anticancer agents: successes in clinical trials and therapeutic application. Int. J. Mol. Sci. 19:E263. doi: 10.3390/ijms19010263

Shehzad, A., Lee, J., and Lee, Y. S. (2013). Curcumin in various cancers. Biofactors 39, 56-68. doi: 10.1002/biof.1068

Sikora, E., Scapagnini, G., and Barbagallo, M. (2010). Curcumin, inflammation, ageing and age-related diseases. Immun. Ageing 7:1. doi: 10.1186/17424933-7-1

Soetikno, V., Sari, F. R., Sukumaran, V., Lakshmanan, A. P., Mito, S., Harima, M., et al. (2012). Curcumin prevents diabetic cardiomyopathy in streptozotocininduced diabetic rats: possible involvement of PKC-MAPK signaling pathway. Eur. J. Pharm. Sci. 47, 604-614. doi: 10.1016/j.ejps.2012.04.018

Taylor, R. A., and Leonard, M. C. (2011). Curcumin for inflammatory bowel disease: a review of human studies. Altern. Med. Rev. 16, 152-156.

The International Diabetes Federation [IDF] (2017). International Diabetes Federation., IDF Diabetes Atlas, 8th Edn. Brussels: International Diabetes Federation.

Topcu-Tarladacalisir, Y., Akpolat, M., Uz, Y. H., Kizilay, G., Sapmaz-Metin, M., Cerkezkayabekir, A., et al. (2013). Effects of curcumin on apoptosis and oxidoinflammatory regulation in a rat model of acetic acid-induced colitis: the roles of c-Jun N-terminal kinase and p38 mitogen-activated protein kinase. J. Med. Food 16, 296-305. doi: 10.1089/jmf.2012.2550

Tschope, C., Walther, T., Escher, F., Spillmann, F., Du, J., Altmann, C., et al. (2005). Transgenic activation of the kallikrein-kinin system inhibits intramyocardial inflammation, endothelial dysfunction and oxidative stress in experimental diabetic cardiomyopathy. FASEB J. 19, 2057-2059. doi: 10.1096/fj.05-4095fje

Wang, Y., Zhou, S., Sun, W., Mcclung, K., Pan, Y., Liang, G., et al. (2014). Inhibition of JNK by novel curcumin analog C66 prevents diabetic cardiomyopathy with a preservation of cardiac metallothionein expression. Am. J. Physiol. Endocrinol. Metab. 306, E1239-E1247. doi: 10.1152/ajpendo.00629.2013

Westermann, D., Rutschow, S., Jager, S., Linderer, A., Anker, S., Riad, A., et al. (2007). Contributions of inflammation and cardiac matrix metalloproteinase activity to cardiac failure in diabetic cardiomyopathy: the role of angiotensin type 1 receptor antagonism. Diabetes Metab. Res. Rev. 56, 641-646. doi: 10.2337/ db06-1163

Wright, E. M., Loo, D. D., and Hirayama, B. A. (2011). Biology of human sodium glucose transporters. Physiol. Rev. 91, 733-794. doi: 10.1152/physrev.00055. 2009

Xu, H., Barnes, G. T., Yang, Q., Tan, G., Yang, D., Chou, C. J., et al. (2003). Chronic inflammation in fat plays a crucial role in the development of obesity-related insulin resistance. J. Clin. Invest. 112, 1821-1830. doi: 10.1172/JCI200319451

Yang, Y., Duan, W., Lin, Y., Yi, W., Liang, Z., Yan, J., et al. (2013). SIRT1 activation by curcumin pretreatment attenuates mitochondrial oxidative damage induced 
by myocardial ischemia reperfusion injury. Free Radic. Biol. Med. 65, 667-679. doi: 10.1016/j.freeradbiomed.2013.07.007

Ye, G., Metreveli, N. S., Donthi, R. V., Xia, S., Xu, M., Carlson, E. C., et al. (2004). Catalase protects cardiomyocyte function in models of type 1 and type 2 diabetes. Diabetes Metab. Res. Rev. 53, 1336-1343. doi: 10.2337/diabetes.53.5. 1336

Yekollu, S. K., Thomas, R., and O'sullivan, B. (2011). Targeting curcusomes to inflammatory dendritic cells inhibits NF- $\mathrm{KB}$ and improves insulin resistance in obese mice. Diabetes Metab. Res. Rev. 60, 2928-2938. doi: 10.2337/db11-0275

You, S., Qian, J., Sun, C., Zhang, H., Ye, S., Chen, T., et al. (2018). An Aza resveratrol-chalcone derivative $6 \mathrm{~b}$ protects mice against diabetic cardiomyopathy by alleviating inflammation and oxidative stress. J. Cell Mol. Med. 22, 1931-1943. doi: 10.1111/jcmm.13477

Yu, W., Wu, J., Cai, F., Xiang, J., Zha, W., Fan, D., et al. (2012). Curcumin alleviates diabetic cardiomyopathy in experimental diabetic rats. PLoS One 7:e52013. doi: 10.1371/journal.pone. 0052013
Yu, W., Zha, W., Ke, Z., Min, Q., Li, C., Sun, H., et al. (2016). Curcumin protects neonatal rat cardiomyocytes against high glucose-induced apoptosis via PI3K/Akt signalling pathway. J. Diabetes Res. 2016:4158591. doi: 10.1155/ 2016/4158591

Conflict of Interest Statement: The authors declare that the research was conducted in the absence of any commercial or financial relationships that could be construed as a potential conflict of interest.

Copyright (๑) 2018 Zheng, Cheng, Zheng, Feng and Xiao. This is an open-access article distributed under the terms of the Creative Commons Attribution License (CC BY). The use, distribution or reproduction in other forums is permitted, provided the original author(s) and the copyright owner are credited and that the original publication in this journal is cited, in accordance with accepted academic practice. No use, distribution or reproduction is permitted which does not comply with these terms. 